\title{
Etiological causes of exudative pleural effusion in patients treated in a teaching hospital
}

\author{
PK Chowdhury ${ }^{1}$, S Ahmed ${ }^{2}$, P Biswas ${ }^{3}$, DK Ghosh ${ }^{4}$, SMT Alam ${ }^{5}$, SK Podder 6
}

\begin{abstract}
The aim of this study was to find out the common etiological causes of exudative pleural effusion in patients before starting treatment. Fifty patients, diagnosed with pleural effusion on admission were randomly selected from Medicine and Paediatric wards of Khulna Medical College Hospital during the period from March 2016 to November 2016. Etiological diagnosis was established by sequential clinical history and findings on physical examination, laboratory tests, chest radiograph, CT scan of the chest and pleural fluid analysis. Patients who remained undiagnosed were subjected to fibro-optic bronchoscopy, thoracoscopic pleural biopsy, and histopathology. Among the patients having pleural effusion, there were tuberculosis, pneumonia, malignancy and systemic lupus erythematosus in $27(54 \%), 11(22 \%), 7(14 \%)$ and $1(2 \%)$, respectively. Despite all investigations, $4(8 \%)$ were remained undiagnosed etiologically. Most of the pleural effusion cases were diagnosed as tuberculosis. Early and adequate treatment resulted in complete recovery of the patients.
\end{abstract}

Key words: Etiological causes, pleural effusion, teaching hospital.

\section{Introduction}

Pleural effusion is common in respiratory medicine. It is a serious local or systemic disease and calls for urgent investigations to determine its cause. The patho-physiological mechanisms underlying pleural effusion include an increased pulmonary capillary pressure, decreased plasma oncotic pressure, increased permeability of pleural membrane, mediastinal involvement with reduced pleural lymphatic drainage, bronchial obstruction with high negative intrapleural pressure, and imbalance between formation and absorption of fluid. ${ }^{1,2}$ The effusion occurring through pressure filtration without capillary injury is termed as transudate. Common examples are congestive cardiac failure
(CCF), renal failure, superiorvenacava obstruction, constrictive pericarditis, liver cirrhosis, fluid overload, and hypoalbuminaemia, Meigs' syndrome, etc. ${ }^{3}$ On the other hand, inflammatory fluid leaking between cells due to local factors is termed an exudate, as in bacterial pneumonia, viral infections, tuberculosis, malignancy, subphrenic pathology and Dressler's syndrome. ${ }^{1-4}$ It may be noted that a malignant disease and pulmonary embolism may produce either a transudative or an exudative effusion. Exudates and transudates are best differentiated by Light's three criteria: i) ratio of pleural fluid protein to serum protein $>0.5$, ii) ratio of pleural fluid to the serum lactate dehydrogenase $(\mathrm{LDH})>0.6$ and iii)

1. PK Chowdhury, MBBS, DTCD, Associate Professor, Respiratory Medicine, Khulna Medical College, Khulna

2. S Ahmed, MBBS, MPhil, Associate Professor, Radiology \& Imaging, Kustia Medical College, Kushtia

3. P Biswas, MBBs, Assistant Registrar, Paediatrics, Khulna Medical College Hospital, Khulna

4. DK Ghosh, MBBS, MD, FCPS, Assistant Professor, Endocrinology, Khulna Medical College, Khulna

5. SMT Alam, MBBS, MD, Medical Officer, Transfution Medicine, Khulna Medical College Hospital, Khulna

6. SK Padder, MBBS, Assistant Registrar, Khulna Medical College Hospital, Khulna 
absolute value of pleural fluid LDH >twothirds of the upper normal limit for serum. While exudates meet one or more of the three criteria, transudates meet none. ${ }^{1}$ Ninety percent cases of pleural effusion in the western countries have been reported to be the result from only five diseases: CCF, pneumonia, malignancy, pulmonary embolism, and viral infections. Twenty to forty percent of hospitalized patients with bacterial pneumonia develop pleural effusion. ${ }^{1-5}$ Pleural effusion is a significant respiratory problem that needs hospitalization.
The present study was done to evaluate the common causes of pleural effusion in a developing country and also to compare that of developed countries.

\section{Materials and method}

This prospective observational study was conducted in medicine and paediatric wards of Khulna Medical College Hospital, Khulna over a period of nine months from March 2016 to November 2016. During this period 50 patients were admitted in medicine ward and diagnosed as pleural effusion based on

Table 1. Age and gender distribution of the patients with pleural effusion

\begin{tabular}{crrr}
\hline Age in years & Male & Female & Total (\%) \\
\hline $10-19$ & 2 & 1 & $3(6)$ \\
$20-29$ & 9 & 3 & $12(24)$ \\
$30-39$ & 10 & 4 & $14(28)$ \\
$40-49$ & 11 & 4 & $15(30)$ \\
$50-59$ & 4 & 2 & $6(12)$ \\
\hline Total & 36 & 14 & $50(100)$ \\
\hline
\end{tabular}

Table 2. Findings of pleural fluid study

\begin{tabular}{lrc}
\hline Physical color & $\begin{array}{r}\text { Number of } \\
\text { patients (\%) }\end{array}$ & $\begin{array}{r}\text { Predominant } \\
\text { cell count }\end{array}$ \\
\hline Straw & $32(64)$ & Lymphocyte \\
Haemorrhagic & $7(14)$ & Lymphocyte \\
Turbid & $11(22)$ & Neutrophil \\
\hline Total & $50(100)$ & \\
\hline
\end{tabular}

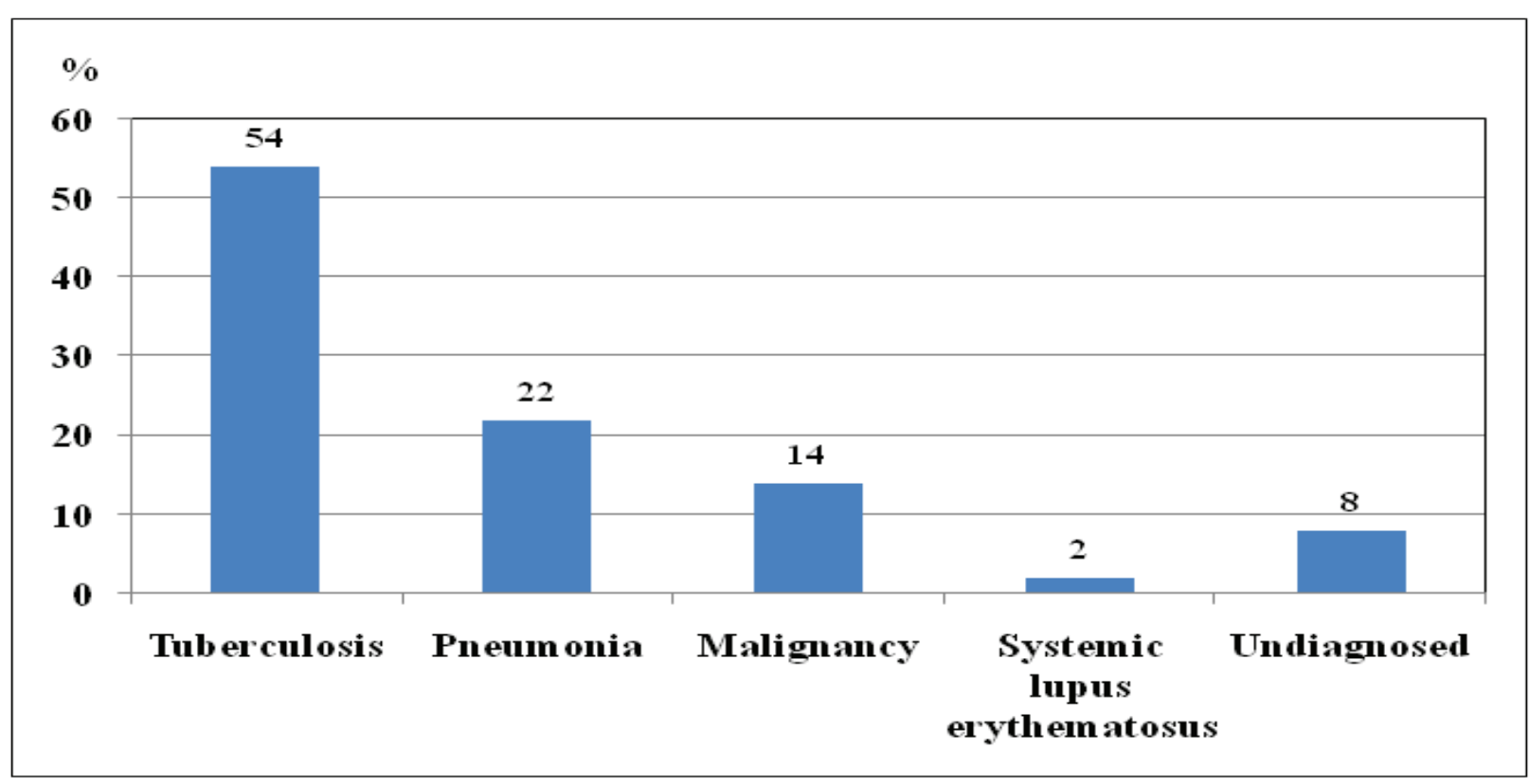

Fig. 1. Etiology of exudative pleural effusion. 
comprehensive clinical history, complete physical examination, a chest $\mathrm{X}$-ray and diagnostic ultrasonography. Subjects of either sex over 10 years of age were included in this study. Subjects $<10$ years of age were excluded from this study.

Complete blood count (CBC) with ESR, chest radiograph and pleural fluid study for biochemical, cytological, gram staining and malignant cells, serum protein and sputum examinations for acid fast bacillus (AFB) were done in all cases. Ultrasonography of the whole abdomen and serological test (ANA test) were done in some cases. Thoracocentesis was done in all cases for pleural fluid analysis. Pleural fluid was examined for biochemical, bacteriological and cytological list.

Inconclusive cases were evaluated by following investigation: fiber-optic bronchoscopy, broncho-alveolar lavage, fine needle aspiration cytology (FNAC) and gene expert. Pleural biopsy, AFB culture and pleural fluid Adenosine Deaminase were not done due to non availability.

\section{Results}

Table 1 shows the age and gender distribution of study subjects. There were 36 male and 14 female patients participated in the study. Most of them (88\%) were of the age range between 20 to 50 years.

Fig. 1 depicts the etiology of exudative pleural effusion, where tuberculosis, pneumonia, malignancy, systemic lupus erythematosus and inconclusive cases were $27(54 \%), 11$ $(22 \%), 7(14 \%), 1(2 \%)$ and $4(8 \%)$, respectively. Among tuberculosis cases, $20(40 \%)$ were sputum positive for AFB and 7 (14\%) were diagnosed by gene expert for tuberculosis. The malignant pleural effusion cases, 7 $(14 \%)$, were confirmed by FNAC of lymphnode, lung biopsy, fiber-optic broncoscopy for bronco-alveolar lavage examination and bronchial tissue biopsy for histopathological examination revealing 5 cases as adenocarcinoma of lung and 2 cases as squamous cell carcinoma.

Table 2 shows the physical characteristics and cytology of pleural fluid. The color of the fluids was straw (64\%), turbid (22\%) and haemorrhagic $(14 \%)$. The cytology was lymphocyte (78\%) and neutrophil (22\%) predominant.

\section{Discussion}

This prospective observational study was conducted among 50 patients of pleural effusion with aim to determine the etiology. Out of 50 patients 36 (72\%) were males, whereas 14 (28\%) were females. In a study of developed country, cause of pleural effusion were malignancy $(24 \%)$, parapneumonia $(22 \%)$, tuberculosis(14\%), hemothorax $(4 \%)$, liver cirrhosis $(2 \%)$ indicating malignancy was the common cause of pleural effusion but in our study common cause of pleural effusion is tuberculosis which does not correlate. ${ }^{1}$ In another study of Hyderabad, Pakistan, common cause of pleural effusion was tuberculosis $(66 \%)$ which supports our study. ${ }^{2}$ Different studies show that tuberculosis is the majority cause of pleural effusion in developing country and our study correlate with other study. Out of 50 pleural effusion cases; right sided pleural effusion $33(66 \%)$, left sided pleural effusion $17(34 \%)$. Study of exudative pleural effusion reveals sputum for AFB positive 20 (40\%), FNAC 9 (18\%), fiber-optic bronchoscopy positive $2(4 \%)$, gene expert for tuberculosis positive in 7 (14\%). But 4 (8\%) cases were undiagnosed. In a study of a developed country the cause of $15 \%$ to $20 \%$ of all pleural effusions remained unknown despite intensive diagnostic efforts. ${ }^{1}$

The present study correlate with other studies that right sided pleural effusion is more common than left sided pleural effusion. Majority causes of exudative pleural effusions were tuberculosis, pneumonia, malignancy, which is almost similar to the findings of other studies in developing countries. ${ }^{2}$ But in developed country scenario is different where malignancy is in the $1^{\text {st }}$ position then parapnumonic effusion, systemic lupus erythematosus and other exudative causes but tuberculosis is almost nil.

\section{Conclusion}

In developed countries, the common cause of pleural effusion are pneumonia and malignancy but in developing country the common cause of pleural effusion is tuberculosis due to poverty, overcrowding 
and inadequate treatment. Early and adequate treatment results in complete recovery of the patient. Otherwise multi-drugs resistant tuberculosis may develop.

\section{References}

1. Maskell NA, Butland RJ. Pleural diseases group: standards of care committee, British Thoracic Society (BTS) guidelines for the investigation of a unilateral pleural effusion in adults. Thorax 2003;58:8-17.

2. Memon SAB, Shaikh SJ. The etiology of pleural effusion in children: Hyderabad experience. Pak J Med Sci 2007;23:86-7.

3. Mehta AA, Patel MN, Soni $A H$, et al. Investigation into role of medical pleuroscopy in the diagnosis and management of patients with pleural diseases. Indian J Thorac Cardiovasc Surg 2012;28:120-6.

4. Beenish A, Kanza I, Khawaja TM. Management of tuberculous pleural effusion. J Biomed Sci Res 2011;3:302-7.

5. Procel JM, Light RW. Diagnostic approach to pleural effusion in adults. Am Fam Physician 2006; 73(7):1211-20.

\section{Suggestion for citation of the above:}

Chowdhury PK, Ahmed S, Biswas P, Ghosh DK, Alam SMT, Podder SK. Etiological causes of exudative pleural effusion in patients treated in a teaching hospital. Mediscope 2017;4(1):30-3. 